\title{
30. A MAGNETO-MINERALOGICAL STUDY OF BASALT SAMPLES FROM HOLES 519A, 522B, AND 524, LEG 73 OF THE DEEP SEA DRILLING PROJECT ${ }^{1}$
}

\author{
J. Housden, Department of Geophysics and Planetary Physics, University of Newcastle upon Tyne, \\ Newcastle upon Tyne, United Kingdom \\ P. Tucker, ${ }^{2}$ Department of Geophysics, University of Edinburgh, Mayfield Road, Edinburgh, United Kingdom \\ and \\ W. O'Reilly, Department of Geophysics and Planetary Physics, University of Newcastle upon Tyne, \\ Newcastle upon Tyne, United Kingdom
}

\begin{abstract}
The basalts in Holes 519A, 522B, and 524 were studied for intensity of natural remanent magnetization, magnetic hysteresis, magnetic susceptibility, stability of isothermal remanence, and thermomagnetic behavior. Some of these properties are sensitive to both the composition and the microstructure of the magnetic minerals, others to composition only. Thus it is possible to separate the two effects and to trace the variation of effective magnetic grain size and degree of alteration within a lithologic unit or over a yet larger distance or time interval. The flow in Hole 519A is highly maghemitized at the top, the degree of maghemitization decreasing with depth in the flow. Effective grain size increases with increasing depth. Electron microprobe analysis of the titanomaghemite grains in these samples provides no support for the leaching out of iron during alteration. The pillows and flows in Hole 522B are distributed among a number of cooling units, and no systematic downhole variations are apparent. The inferred magneto-petrology is consistent with the cooling and alteration history that might be expected within the units. The upper and lower sills in Hole 524 are more uniform and have a larger concentration of well-developed magnetic mineral grains than the pillows and flows in Holes 519A and 522B. Maghemitization appears to have developed from the boundaries of the sills that are in contact with the sediments between the sills.
\end{abstract}

\section{INTRODUCTION}

The measurement of the magnetic properties of rocks gives information about the composition, concentration, and microstructure (e.g., grain size) of the magnetic mineral fraction. This information is needed for the investigation of problems related to magnetization both directly (the magnetic history of the rocks and anomalies in the geomagnetic field) and indirectly (problems in geochemistry and geophysics). The remanence directions of rocks from submarine basement, for example, are used to infer spreading rates, and the intensities of magnetization are used to model anomalies in the geomagnetic field and to estimate the thickness of the magnetized layer. In many cases, the effects of thermal and.chemical history after the acquisition of primary remanence must also be assessed (see Ryall et al., 1977): an understanding of the post-deuteric alteration of the magnetic minerals may elucidate the general geochemistry of the crust/ocean interface or cast light on local geophysical problems, such as the heat flow in the submarine crust.

In the present study we examine variations in magnetic properties and magnetic mineralogy on both a small scale (within a given lithologic unit) and on a larger scale (within the depth of the basement penetrated or over the distance between the drill site and the spreading center). We make use of both the measurement of magnetic prop-

\footnotetext{
${ }^{1}$ Hsï, K. J., LaBrecque, J. L., et al., Init. Repts. DSDP, 73: Washington (U.S. Govt. Printing Office).

2 Present address: Warren Spring Laboratory, Gunnels Wood Road, Stevenage, Hertfordshire, United Kingdom
}

erties, such as magnetic hysteresis parameters and the variation of high-field magnetization with temperature, and other analytical methods (such as optical microscopy and electron probe microanalysis). This report presents results for minicore samples acquired from a flow in Hole 519A, from pillows and flows in Hole 522B, and from sills in Hole 524.

\section{FLOW OF HOLE 519A}

\section{Optical and Electron Microprobe Analysis}

The results of optical examination of Lithologic Unit 4 in Hole 519A are summarized in Table 1. The concentration of opaques has been roughly estimated by eye, and this in turn has been subdivided into fractions of titanomagnetite, ilmenite, pyrite, limonite, and sulfide spherules. Rough visual estimates of the mean grain sizes and size ranges have also been made for the opaques. Both the matrix grain size and the size of the magnetic opaques (titanomagnetites) increase with increasing depth in the flow.

Titanomagnetites are by far the most abundant of the opaques. In the finer grain specimens (Samples 519A-9-1, $11 \mathrm{~cm}$ and 519A-9-1, $27 \mathrm{~cm}$ ) they are skeletal to subhedral, appear unaltered, and frequently occur in chain structures. In the coarser samples they exhibit a texture that may indicate low-temperature alteration. Curvilinear (conchoidal) shrinkage cracks are also apparent. The apparent alteration generally becomes more pronounced with increasing depth in the flows. Only one grain was found (in Sample 519A-9-4, $84 \mathrm{~cm}$ ) with the intergrown lamellae associated with high-temperature 
Table 1. Optical properties of opaque minerals.

\begin{tabular}{|c|c|c|c|c|c|c|c|}
\hline $\begin{array}{l}\text { Core-Section } \\
\text { (level in } \mathrm{cm} \text { ) }\end{array}$ & $\begin{array}{l}\text { Proportion of } \\
\text { whole rock } \\
(\%)\end{array}$ & Mineral $^{\mathrm{a}}$ & $\begin{array}{c}\text { Proportion } \\
\text { of opaques } \\
(\%)\end{array}$ & $\begin{array}{c}\text { Grain } \\
\text { size range } \\
(\mu \mathrm{m})\end{array}$ & $\begin{array}{c}\text { Mean } \\
\text { grain size } \\
(\mu \mathrm{m})\end{array}$ & $\begin{array}{l}\text { Grain } \\
\text { form }\end{array}$ & Basalt type \\
\hline \multicolumn{8}{|l|}{ Hole 519A } \\
\hline $9-1,11$ & 5 & $\begin{array}{l}\mathrm{Tm} \\
\mathrm{Li}\end{array}$ & $\begin{array}{r}99.5 \\
0.5\end{array}$ & $\begin{array}{r}0-10 \\
20-60\end{array}$ & $\begin{array}{l}2.5 \\
40\end{array}$ & $\mathrm{Sk} / \mathrm{Su}$ & \multirow{7}{*}{ Flow } \\
\hline $9-1,27$ & $<5$ & $\mathrm{Tm}$ & $\begin{array}{r}99 \\
1\end{array}$ & $\overline{-}$ & $\begin{array}{l}3 \\
3.5\end{array}$ & $\mathrm{Sk} / \mathrm{Su}$ & \\
\hline $9-1,80$ & 5 & $\begin{array}{l}\mathrm{Tm} \\
\text { Il } \\
\mathrm{Li} \\
\mathrm{S}\end{array}$ & $\begin{array}{l}98 \\
2 \\
\text { (c) } \\
\text { (d) }\end{array}$ & $\begin{array}{l}0-12 \\
0-40 \\
- \\
-\end{array}$ & $\frac{3.5}{3.5}$ & $\begin{array}{l}\text { Patches } \\
\text { Laths }\end{array}$ & \\
\hline $9-2,29$ & 5 & $\begin{array}{l}\mathrm{Tm} \\
\text { Il } \\
\mathbf{P y} \\
\mathbf{L i}\end{array}$ & $\begin{array}{l}77 \\
20 \\
\text { (c) } \\
3\end{array}$ & $\begin{array}{r}0-120 \\
0-120 \\
80,20 \\
40-150\end{array}$ & $\begin{array}{l}40 \\
20 \\
\frac{60}{-}\end{array}$ & Laths & \\
\hline $9-3,145$ & 6 & $\begin{array}{l}\text { Tm } \\
\mathrm{S}\end{array}$ & $\begin{array}{r}99 \\
1\end{array}$ & $\begin{array}{l}0-70 \\
0-5\end{array}$ & $\begin{array}{r}20 \\
3\end{array}$ & Skeletal laths & \\
\hline $9-4,40$ & $>5$ & $\begin{array}{l}\text { Tm } \\
\text { Il } \\
\text { Py } \\
\text { S }\end{array}$ & $\begin{array}{r}73 \\
15 \\
2 \\
10\end{array}$ & $\begin{array}{c}5-160 \\
0-200 \\
10-60 \\
5-40\end{array}$ & $\begin{array}{l}80 \\
40 \\
20 \\
10\end{array}$ & $\begin{array}{r}\text { Laths } \\
\text { - } \\
-\end{array}$ & \\
\hline.$\quad \therefore$ & 7 & $\begin{array}{l}\text { Tm } \\
\text { Il } \\
\text { Py } \\
\text { S }\end{array}$ & $\begin{array}{r}84 \\
10 \\
1 \\
5\end{array}$ & $\begin{array}{c}5-350 \\
0-200 \\
0.5-20 \\
0.5-40\end{array}$ & $\begin{array}{r}80 \\
50 \\
5 \\
10\end{array}$ & $\begin{array}{l}\text { Sk/An } \\
\text { Laths } \\
=\end{array}$ & \\
\hline \multicolumn{8}{|l|}{ Hole 522B } \\
\hline $3-4,61$ & 3 & $\begin{array}{l}\text { Tm } \\
\text { Py }\end{array}$ & $\begin{array}{r}99.5 \\
0.5\end{array}$ & $\begin{array}{l}0-6 \\
0-5\end{array}$ & $\begin{array}{l}2 \\
2\end{array}$ & $\begin{array}{l}\mathrm{Su} / \mathrm{An} \\
\mathrm{Su}\end{array}$ & Pillows \\
\hline $4-1,17$ & 4 & $\begin{array}{l}\text { Tm } \\
\text { Py }\end{array}$ & $\begin{array}{r}99 \\
1\end{array}$ & $\begin{array}{l}0-75 \\
0-15\end{array}$ & $\begin{array}{r}10 \\
2\end{array}$ & $\begin{array}{l}\text { Sk/An } \\
\text { Su/Eu }\end{array}$ & \multirow{3}{*}{ Flow } \\
\hline $4-1,43$ & 4 & $\begin{array}{l}\text { Tm } \\
\text { Py }\end{array}$ & $\begin{array}{r}99.5 \\
0.5\end{array}$ & $\begin{array}{l}0-60 \\
-\end{array}$ & $\begin{array}{r}15 \\
3\end{array}$ & $\begin{array}{l}\text { Su/Sk } \\
\text { Su/Sk }\end{array}$ & \\
\hline $5-2,30$ & 2 & $\mathrm{Tm}$ & 100 & $0-3$ & $<1$ & $\mathrm{An} / \mathrm{Su}$ & \\
\hline $5-2,75$ & 3 & $\begin{array}{l}\text { Tm } \\
\text { Py } \\
\text { S }\end{array}$ & $\begin{array}{r}99.8 \\
\text { (e) } \\
0.2\end{array}$ & $\begin{array}{l}0-25 \\
0-6 \\
-\end{array}$ & $\begin{array}{l}3 \\
4 \\
1\end{array}$ & $\begin{array}{l}\mathrm{Sk} / \mathrm{Su} \\
\mathrm{Sk}\end{array}$ & \multirow[t]{2}{*}{ Flow } \\
\hline $5-2,139$ & 4 & $\begin{array}{l}\text { Tm } \\
P y\end{array}$ & $\begin{array}{r}99 \\
1\end{array}$ & $\begin{array}{l}0-40 \\
0-20\end{array}$ & $\begin{array}{r}15 \\
2\end{array}$ & $\begin{array}{l}\mathrm{Sk} / \mathrm{Su} \\
\mathrm{Su}\end{array}$ & \\
\hline $5-3,41$ & 3 & $\mathrm{Tm}$ & 100 & $30^{\mathrm{f}}$ & 7 & Sk & Flow/sill? \\
\hline \multicolumn{8}{|l|}{ Hole 524} \\
\hline $29-2,28$ & 5 & $\begin{array}{l}\text { Tm } \\
\text { II } \\
\text { Py }\end{array}$ & $\begin{array}{r}55 \\
40 \\
1\end{array}$ & $\begin{array}{l}10-100 \\
10-100 \\
30-150\end{array}$ & $\begin{array}{l}20 \\
20 \\
30\end{array}$ & $\begin{array}{l}\mathrm{Su} / \mathrm{Eu} \\
\mathrm{Laths,} \mathrm{Su} / \mathrm{Sk} \\
\mathrm{Eu} / \mathrm{Su}\end{array}$ & \multirow{5}{*}{ Upper sill } \\
\hline $30-1,141$ & 4 & $\begin{array}{l}\text { Tm } \\
\text { Il } \\
\text { Py }\end{array}$ & $\begin{array}{r}65 \\
30 \\
5\end{array}$ & $\begin{array}{c}20-100 \\
5-75 \\
0-100\end{array}$ & $\begin{array}{r}40 \\
30 \\
5\end{array}$ & $\begin{array}{l}\mathrm{Eu} / \mathrm{Sk} \\
\text { Laths, Eu/Su } \\
\text { Su }\end{array}$ & \\
\hline $30-2,52$ & 4 & $\begin{array}{l}\text { Tm } \\
\text { Il } \\
\text { Py }\end{array}$ & $\begin{array}{r}75(55) \\
25(15) \\
0(30)\end{array}$ & $\begin{array}{r}0-180 \\
0-230 \\
50-300\end{array}$ & $\begin{array}{r}20 \\
75 \\
200\end{array}$ & $\begin{array}{l}\mathrm{Eu} / \mathrm{Su} \\
\mathrm{Laths,} \mathrm{Eu/Su} \\
\text { Eu/spherical }\end{array}$ & \\
\hline $30-2,110$ & 5 & $\begin{array}{l}\text { Tm } \\
\text { Il } \\
\text { Py }\end{array}$ & $\begin{array}{r}(78) \\
(15) \\
(7)\end{array}$ & $\begin{array}{c}0-60 \\
- \\
20-400\end{array}$ & $\frac{30}{100}$ & $\begin{array}{l}\mathrm{Eu} / \mathrm{Sk} \\
\mathrm{Laths,} \mathrm{Eu} / \mathrm{Su} \\
\mathrm{Eu} / \mathrm{Su}\end{array}$ & \\
\hline $31-2,69$ & 6 & $\begin{array}{l}\text { Tm } \\
\text { Il }\end{array}$ & $\begin{array}{l}80 \\
20\end{array}$ & $\begin{array}{l}1-100 \\
0-200\end{array}$ & $\begin{array}{l}70 \\
35\end{array}$ & $\begin{array}{l}\text { Eu/Sk } \\
\text { Laths, }{ }^{\mathrm{g}} \mathrm{Su}\end{array}$ & \\
\hline $33-2,52$ & 4 & $\begin{array}{l}\text { Tm } \\
\text { Il } \\
\text { Py }\end{array}$ & $\begin{array}{l}90 \\
10 \\
\text { (d) }\end{array}$ & $\begin{array}{r}50-150 \\
40-100 \\
500\end{array}$ & $\begin{array}{r}100 \\
70 \\
5\end{array}$ & $\begin{array}{l}\text { Eu/Sk } \\
\text { Laths } \\
\text { Eu }\end{array}$ & \multirow{5}{*}{$\begin{array}{l}\text { Lower sill, } \\
\text { central part }\end{array}$} \\
\hline $33-2,98$ & 5 & $\begin{array}{l}\text { Tm } \\
\text { Py }\end{array}$ & $\begin{array}{r}100(70) \\
0(30)\end{array}$ & $\begin{array}{l}20-100 \\
50-500\end{array}$ & $\begin{array}{r}50 \\
200\end{array}$ & $\begin{array}{l}\mathrm{Su} / \mathrm{Sk} \\
\mathrm{Eu} / \mathrm{Su} / \mathrm{Sk}\end{array}$ & \\
\hline $33-3,29$ & 5 & $\mathrm{Tm}$ & 100 & $20-250$ & 150 & $\mathrm{Eu} / \mathrm{Sk}$ & \\
\hline $33-3,100$ & 6 & $\mathrm{Tm}$ & 100 & $50-300$ & 175 & $\mathrm{Eu} / \mathrm{Su} / \mathrm{Sk}$ & \\
\hline $33-3,131$ & 6 & $\mathrm{Tm}$ & 100 & $20-200$ & 75 & $\mathrm{Eu} / \mathrm{Sk}$ & \\
\hline
\end{tabular}

Note: For each hole, samples are listed in order of increasing sub-bottom depth. Mineral compositions given in parentheses include veins.

a $\mathrm{Tm}=$ titanomagnetite, $\mathrm{Il}=$ ilmenite, $\mathrm{Py}=$ pyrite, $\mathrm{Li}=$ limonite, $\mathrm{S}=$ sulfide spherules.

$\mathrm{b} \mathrm{An}=$ anhedral, $\mathrm{Eu}=$ euhedral, $\mathrm{Sk}=$ skeletal, $\mathrm{Su}=$ subhedral. Patches $=$ granular conglomerates.

$\begin{array}{ll}\text { c Two occurrences. } & \text { f Around vein. } \\ \text { d One occurrence. } & \text { g Rare. }\end{array}$ 
(deuteric) oxidation. In this unusually large grain $(200 \times$ $200 \mu \mathrm{m})$, ilmenite lamellae all follow the same plane. Two shrinkage cracks also penetrate the grain, and although they may be presumed to have formed after the deuteric alteration, the sequence of events is not clear cut. The skeletal/anhedral form of the titanomagnetites, in combination with their basaltic glass matrix, shows that the grains formed late in the sequence of crystallization.

No ilmenite was revealed by optical or microprobe examination in Sample 519A-9-1, $11 \mathrm{~cm}$ or 519A-9-1, 27 $\mathrm{cm}$. However, two of eight small grains chosen at random from Sample 519A-9-1, $80 \mathrm{~cm}$ were ilmenite, and grains of ilmenite, which could be identified under reflected light, were abundant among the larger grains. Ilmenite is commonly more euhedral than the titanomagnetites and tends to form laths in the coarser specimens. These lath-shaped grains are more homogeneous than the smaller prismatic ilmenites, which show a degree of variation similar to that of titanomagnetite in any given specimen. This anomalous homogeneity of the larger ilmenites suggests two phases of ilmenite growth, although there is no other supporting evidence.

Sulfides occur in two forms. Subhedral pyrite occurs mainly in microfractures, but far more common are composite sulfide spherules, which become increasingly more abundant with increasing depth. The boundaries between the components of the spherules occasionally show a perlitic texture. Three spherules were probed for $\mathrm{Fe}, \mathrm{S}, \mathrm{Cu}, \mathrm{Zn}, \mathrm{Pb}, \mathrm{Cr}, \mathrm{Al}, \mathrm{Mg}, \mathrm{Si}, \mathrm{Mn}$, and $\mathrm{Ti} . \mathrm{Fe}, \mathrm{S}$, and $\mathrm{Cu}$ were found to be present, as were small amounts of $\mathrm{Al}, \mathrm{Mg}, \mathrm{Si}$, and $\mathrm{Mn}$. One possible explanation for the presence of $\mathrm{Si}$ is that a silicate has filled the curvilinear cracks between the components of the spherule. A Sideficient rim in one spherule may indicate a subsequent migration of $\mathrm{Si}$. Two spherules in Sample 519A-9-4, $84 \mathrm{~cm}$ were found to have areas with high concentrations of $\mathrm{Fe}$ and $\mathrm{Cu}$. The iron-rich areas were identified as pyrrhotite. However, the composition may be near to troilite (FeS); if so, these areas may not influence magnetic properties.

Limonite $\left(\mathrm{FeO} \cdot \mathrm{OHnH}_{2} \mathrm{O}\right)$ occurs in botryoidal, anhedral, and euhedral forms (the latter probably pseudomorphed oxide), often in large masses that exhibit a cloth texture. In three instances a gray silicate mineral (possibly zeolites) apparently replaced the limonite.

The oxides were probed for $\mathrm{Fe}, \mathrm{Ti}, \mathrm{Mn}, \mathrm{Mg}, \mathrm{Al}, \mathrm{Si}$, $\mathrm{Ta}, \mathrm{Nb}, \mathrm{Ca}, \mathrm{Ni}, \mathrm{Zn}, \mathrm{Cr}$, and V. Four 10-s counts were recorded for each element on each point, and metal standards were referred to before and after each grain analysis. The apparent presence of Si may be due to beam spreading into the surrounding matrix, or (in the case of larger grains) the presence of silicate inclusions or silicates in shrinkage cracks. If either hypothesis is true, the $\mathrm{Mg}$ and $\mathrm{Al}$ analyses will also fail to represent true concentrations for the oxide phase. However, as a working hypothesis all elements analyzed for the titanomagnetites/titanomaghemites were taken as being incorporated in the structure. The composition may then be represented as follows:

$$
\mathrm{Fe}_{(3-x-\alpha-\delta) R} M_{\alpha R}^{2+} M_{\delta R}^{3+} \mathrm{Ti}_{(x-\beta) R}^{4+} M_{\beta R}^{4+} \square_{3(1-R)} \mathrm{O}_{4}
$$

where $M^{2+}, M^{3+}$, and $M^{4+}$ represent $(\mathrm{Mn}+\mathrm{Mg}), \mathrm{Al}$, and $\overline{\mathrm{Si}}$, respectively. This compound can be considered as having been derived by substitution and the addition of oxygen to $\mathrm{Fe}_{3-x} \mathrm{Ti}_{x} \mathrm{O}_{4}$. $R$ expresses the deviation of the composition from that appropriate to a stoichiometric spinel. The number of cations per spinel formula unit (four oxygens) is $3 R$. The number of vacant lattice sites, $\square$, per formula unit is $3(1-R)$. A second primary composition parameter, $z$ (the oxidation parameter), also describes the deviation from stoichiometry in the case of a titanomagnetite/titanomaghemite. This is related to $R$ by $R=8 /[8+z(1-x-\alpha)]$.

The results of the microprobe analyses for the samples from Hole 519A are listed in Table 2. Oxygen was determined by difference from the weight percent of the metals returned by the probe. The weight percent of all the elements was converted to atomic proportions and normalized to four oxygens. These normalized atomic proportions are listed in the table. The sum of the elements is $3 R$. $x$ and $z$ are then determined from the analyses, according to the above formula unit. The values of $x$, the determination of which depends only on the metal analyses, is relatively accurate, as the scatter in the values for a given sample reveals (some of the scatter no doubt being due to real variations in chemical composition within a sample). The determination of $z$ is much less accurate, because the uncertainties in the metal analyses appear several times (in nested summations) in the expression for the error in $z$. There may also be systematic errors (e.g., errors due to beam spreading) that lead to a deficiency in metals that would produce systematically high calculated $z$ values. The scatter in determined $z$ values is therefore much higher, even within a given grain. Inhomogeneity in degree of alteration (as indeed is suggested by X-ray distribution images for $\mathrm{Fe}$ ) could, of course, account for part of the scatter. Composition does seem to vary with increasing depth in the flow, the mean value of $x$ for each sample, $\bar{x}$, if nothing else. A decrease in $\bar{z}$ with increasing depth is suggested by the data but cannot be taken as definite because of the scatter in the data.

\section{Intensity of Natural Remanent Magnetization and Isothermal Remanent Magnetization}

The intensities of natural remanent magnetization (NRM) were measured by using a spinner magnetometer. The results are summarized in Table 3. Samples were also magnetized to saturation in an electromagnet to produce a saturation remanence $\left(M_{R S}\right)$. This remanence was in turn demagnetized by alternating field, and the median destructive field $(M D F)$ at which the remanence was reduced to one-half its initial value is listed in the table. The intensities of $N R M$ and $M_{R S}$ depend on many parameters-concentration, composition, microstructure, and (in the case of NRM) the strength of the inducing field. No great or systematic variation is obvious within the flow. The ratio $N R M / M_{R S}$ may increase with depth, but more data would be needed to establish 
Table 2. Microprobe analyses of titanomagnetite/titanomaghemite grains.

\begin{tabular}{|c|c|c|c|c|c|c|c|c|c|c|c|c|}
\hline $\begin{array}{l}\text { Core-Sectio } \\
\text { (level in cm }\end{array}$ & & Grain & $\mathrm{Fe}$ & $\mathrm{Ti}$ & $\mathrm{Mn}$ & $\mathrm{Si}$ & Al & Mg & $x$ & $z$ & $\bar{x}$ & $\bar{z}$ \\
\hline \multicolumn{2}{|l|}{ Hole 519A } & & & . 4 & & & & $\therefore$ & & & & \\
\hline \multirow{2}{*}{\multicolumn{2}{|c|}{$9-1,11$}} & 1 & 1.897 & 0.484 & 0.012 & 0.023 & 0.096 & 0.065 & 0.56 & 0.89 & $0.52(0.04)$ & $0.89(0.20)$ \\
\hline & & 2 & 1.594 & 0.499 & 0.015 & 0.310 & 0.137 & 0.187 & 0.55 & 0.57 & $\ldots$ & \\
\hline \multirow{2}{*}{\multicolumn{2}{|c|}{ 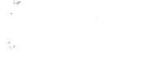 }} & 3 & 1.590 & 0.381 & 0.014 & 0.390 & 0.122 & 0.023 & 0.45 & 1.08 & $\cdots$ & \\
\hline & & 4 & 1.496 & 0.430 & 0.017 & 0.428 & 0.135 & 0.022 & 0.51 & 1.02 & & , \\
\hline \multirow{4}{*}{\multicolumn{2}{|c|}{$9-1,27$}} & 1 & 1.926 & 0.429 & 0.012 & 0.051 & 0.081 & 0.053 & 0.50 & 0.98 & $0.53(0.03)$ & $0.69(0.21)$ \\
\hline & & 2 & 2.110 & 0.492 & 0.013 & 0.042 & 0.080 & 0.074 & 0.53 & 0.37 & & \\
\hline & & 3 & 1.959 & 0.463 & 0.018 & 0.018 & 0.092 & 0.038 & 0.54 & 0.86 & & \\
\hline & & $\begin{array}{l}4 \\
5\end{array}$ & $\begin{array}{l}2.083 \\
2.012\end{array}$ & $\begin{array}{l}0.445 \\
0.517\end{array}$ & $\begin{array}{l}0.012 \\
0.012\end{array}$ & 0.010 & 0.078 & 0.056 & $\begin{array}{l}0.50 \\
0.58\end{array}$ & $\begin{array}{l}0.66 \\
0.60\end{array}$ & & \\
\hline \multirow{5}{*}{\multicolumn{2}{|c|}{$9-1,80$}} & 1 & 2.970 & 0.771 & 0.027 & 0.035 & 0.087 & 0.030 & 0.59 & -1.21 & $0.58(0.03)$ & $0.52(0.89)$ \\
\hline & & 2 & 1.925 & $0.451:$ & 0.018 & 0.026 & 0.065 & 0.010 & 0.54 & 1.07 & & \\
\hline & & 3 & 1.837 & 0.464 & 0.033 & 0.031 & 0.023 & 0.037 & 0.57 & 1.27 & & \\
\hline & & 4 & 1.950 & 0.485 & 0.014 & 0.018 & 0.088 & 0.032 & 0.56 & 0.85 & & \\
\hline & & 5 & 1.925 & 0.565 & 0.016 & 0.014 & 0.104 & 0.053 & 0.63 & 0.62 & & \\
\hline $9-2,29$ & & 1 & 1.891 & 0.516 & 0.008 & 0.056 & 0.056 & 0.050 & 0.60 & 0.86 & $0.58(0.02)$ & $0.52(0.47)$ \\
\hline & & 2 & 2.241 & 0.537 & 0.009 & 0.004 & 0.057 & 0.048 & 0.56 & 0.19 & & \\
\hline $9-3,145$ & & 1 & 1.920 & 0.603 & 0.018 & 0.022 & 0.072 & 0.035 & 0.68 & 0.61 & $0.67(0.07)$ & $0.80(0.21)$ \\
\hline & & 2 & 2.130 & 0.488 & 0.017 & 0.008 & 0.078 & 0.029 & 0.53 & 0.49 & & \\
\hline & & 3 & 1.837 & 0.481 & 0.018 & 0.010 & 0.070 & 0.034 & 0.68 & 0.87 & & \\
\hline & & 4 & 1.729 & 0.611 & 0.015 & 0.027 & 0.060 & 0.039 & 0.74 & 1.00 & & \\
\hline & & 5 & 1.770 & 0.583 & 0.017 & 0.023 & 0.072 & 0.021 & 0.70 & 1.00 & & \\
\hline $9-4,40$ & & 1 & 1.891 & 0.584 & 0.016 & 0.012 & 0.079 & 0.050 & 0.67 & 0.70 & $0.65(0.02)$ & $0.28(0.26)$ \\
\hline & & 2 & 2.097 & 0.618 & 0.013 & 0.006 & 0.089 & 0.086 & 0.64 & 0.16 & & \\
\hline & & 2 & 1.985 & 0.607 & 0.013 & 0.023 & 0.094 & 0.097 & 0.65 & 0.33 & & \\
\hline & & $\begin{array}{l}3 \\
3\end{array}$ & $\begin{array}{l}2.160 \\
2.145\end{array}$ & $\begin{array}{l}0.663 \\
0.657\end{array}$ & $\begin{array}{l}0.013 \\
0.022\end{array}$ & $\begin{array}{l}0.007 \\
0.009\end{array}$ & $\begin{array}{l}0.075 \\
0.082\end{array}$ & $\begin{array}{l}0.073 \\
0.059\end{array}$ & $\begin{array}{l}0.67 \\
0.66\end{array}$ & 0.63 & & \\
\hline & $\rightarrow$ & 4 & 2.145 & 0.631 & 0.011 & 0.007 & 0.090 & 0.077 & 0.64 & 0.07 & & \\
\hline & & 4 & 2.137 & 0.620 & 0.013 & 0.001 & 0.094 & 0.103 & 0.62 & 0.06 & & \\
\hline $9-4,84$ & & 1 & 2.192 & 0.636 & 0.020 & 0.007 & 0.064 & 0.042 & 0.65 & 0.07 & $0.64(0.01)$ & $0.10(0.14)$ \\
\hline & & 1 & 2.087 & 0.617 & 0.019 & 0.006 & 0.060 & 0.045 & 0.65 & 0.30 & & \\
\hline$\therefore$ & & 1 & 2.167 & 0.647 & 0.020 & 0.009 & 0.066 & 0.060 & 0.65 & 0.05 & & \\
\hline & & 1 & 2.185 & 0.649 & 0.020 & 0.007 & 0.064 & 0.046 & 0.65 & 0.05 & & \\
\hline & & 2 & 2.117 & 0.610 & 0.019 & 0.062 & 0.060 & 0.047 & 0.64 & 0.15 & & \\
\hline & & $\begin{array}{l}2 \\
2\end{array}$ & $\begin{array}{l}2.122 \\
2.097\end{array}$ & $\begin{array}{l}0.610 \\
0.612\end{array}$ & $\begin{array}{l}0.019 \\
0.017\end{array}$ & $\begin{array}{l}0.006 \\
0.006\end{array}$ & $\begin{array}{l}0.056 \\
0.069\end{array}$ & $\begin{array}{l}0.054 \\
0.058\end{array}$ & $\begin{array}{l}0.64 \\
0.64\end{array}$ & $\begin{array}{l}0.24 \\
0.25\end{array}$ & & \\
\hline & & 3 & 2.173 & 0.643 & 0.018 & 0.024 & 0.062 & 0.062 & 0.65 & 0.03 & & \\
\hline & & 3 & 2.301 & 0.661 & 0.018 & 0.007 & 0.080 & 0.071 & 0.63 & -0.23 & & \\
\hline & & 3 & 2.152 & 0.631 & 0.015 & 0.007 & 0.068 & 0.068 & 0.64 & 0.10 & & \\
\hline Hole 522B & & & & & & & & & & & & \\
\hline $4-1,17$ & & 1 & 2.096 & 0.538 & 0.021 & 0.027 & 0.078 & 0.032 & 0.58 & 0.39 & $0.62(0.03)$ & $0.59(0.15)$ \\
\hline & & 2 & 2.012 & 0.568 & 0.019 & 0.019 & 0.062 & 0.052 & 0.62 & 0.51 & & \\
\hline & & 3 & 1.952 & 0.557 & 0.018 & 0.016 & 0.049 & 0.030 & 0.64 & 0.73 & & \\
\hline & & 4 & 1.935 & 0.605 & 0.021 & 0.018 & 0.066 & 0.058 & 0.67 & 0.55 & & \\
\hline & & 5 & 2.028 & 0.562 & 0.021 & 0.019 & 0.058 & 0.027 & 0.62 & 0.54 & & \\
\hline & & 6 & 1.869 & 0.522 & 0.020 & 0.050 & 0.068 & 0.066 & 0.60 & 0.83 & & \\
\hline $4-1,43$ & & 1 & 2.205 & 0.652 & 0.021 & 0.155 & 0.085 & 0.103 & 0.61 & -0.370 & $0.60(0.02)$ & $-0.38(0.32)$ \\
\hline & & 2 & 2.270 & 0.628 & 0.018 & 0.122 & 0.124 & 0.032 & 0.59 & -0.317 & & \\
\hline & & 3 & 2.310 & 0.625 & 0.021 & 0.144 & 0.088 & 0.074 & 0.57 & -0.432 & & \\
\hline & & 4 & 2.197 & 0.643 & 0.020 & 0.110 & 0.101 & 0.045 & 0.62 & -0.190 & & \\
\hline & & 5 & 2.398 & 0.707 & 0.020 & 0.331 & 0.127 & 0.129 & 0.57 & -1.017 & & \\
\hline & & 6 & 2.106 & 0.630 & 0.020 & 0.094 & 0.083 & 0.039 & 0.63 & 0.046 & & \\
\hline $5-2,75$ & & 1 & 2.203 & 0.495 & 0.013 & 0.026 & 0.085 & 0.150 & 0.50 . & 0.05 & $0.53(0.03)$ & $0.05(0.01)$ \\
\hline & & 2 & 2.175 & 0.561 & 0.015 & 0.024 & 0.138 & 0.065 & 0.56 & 0.04 & & \\
\hline $5-2,139$ & & 1 & 2.258 & 0.485 & 0.015 & 0.009 & 0.091 & 0.065 & 0.51 & 0.15 & $0.60(0.04)$ & $0.41(0.18)$ \\
\hline$-3, x^{2}$ & & 2 & 2.114 & 0.563 & 0.021 & 0.028 & 0.057 & 0.026 & 0.60 & 0.35 & & \\
\hline & & 3 & 2.103 & 0.572 & 0.023 & 0.019 & 0.073 & 0.088 & 0.62 & 0.41 & & \\
\hline & & 4 & 2.083 & 0.576 & 0.021 & 0.070 & 0.077 & 0.021 & 0.61 & 0.27 & & \\
\hline & & 5 & 1.993 & 0.520 & 0.018 & 0.027 & 0.070 & 0.031 & 0.59 & 0.67 & & \\
\hline & & 6 & 1.970 & 0.574 & 0.021 & 0.021 & 0.062 & 0.031 & 0.64 & 0.60 & & \\
\hline $5-3,41$ & & 1 & 1.744 & 0.453 & 0.017 & 0.123 & 0.098 & 0.073 & 0.54 & 1.10 & $0.58(0.06)$ & $0.69(0.23)$ \\
\hline & & 2 & 1.899 & 0.471 & 0.020 & 0.127 & 0.105 & 0.071 & 0.52 & 0.64 & & \\
\hline 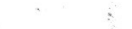 & & 3 & 1.983 & 0.595 & 0.021 & 0.021 & 0.056 & 0.033 & 0.66 & 0.54 & & $\because$ \\
\hline & & 4 & 1.944 & 0.515 & 0.018 & 0.107 & 0.082 & 0.199 & 0.56 & 0.42 & & \\
\hline & & 5 & 1.945 & 0.566 & 0.018 & 0.043 & 0.014 & 0.029 & 0.64 & 0.74 & & \\
\hline Hole 524 & & & & & & & & & & & & $e_{\mathrm{it}}$ \\
\hline $29-2,28$ & & 1 & 2.073 & 0.545 & 0.015 & 0.006 & 0.151 & 0.179 & 0.55 & 0.06 & $0.59(0.04)$ & $0.39(0.17)$ \\
\hline & & 2 & 2.096 & 0.538 & 0.019 & 0.008 & 0.027 & 0.046 & 0.59 & 0.51 & & \\
\hline & & 3 & 2.001 & 0.618 & 0.023 & 0.008 & 0.091 & 0.089 & 0.66 & 0.31 & & \\
\hline & & 4 & 2.035 & 0.570 & 0.027 & 0.010 & 0.042 & 0.056 & 0.62 & 0.50 & & \\
\hline & & 5 & 2.165 & 0.488 & 0.023 & 0.010 & 0.004 & 0.015 & 0.54 & 0.59 & & . \\
\hline & & 6 & 2.058 & 0.558 & 0.025 & 0.008 & 0.089 & 0.072 & 0.60 & 0.37 & & \\
\hline $30-2,52$ & & 1 & 1.728 & 0.562 & 0.024 & 0.031 & 0.145 & 0.128 & 0.64 & 0.800 & $0.64(0.01)$ & $0.952(0.10)$ \\
\hline & & 2 & 1.740 & 0.547 & 0.031 & 0.025 & 0.137 & 0.094 & 0.64 & 0.886 & & 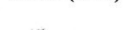 \\
\hline & & 3 & 1.692 & 0.524 & 0.027 & 0.044 & 0.124 & 0.069 & 0.63 & 1.105 & . & $\ldots$ \\
\hline & & 4 & 1.688 & 0.554 & 0.043 & 0.056 & 0.120 & 0.064 & 0.63 & 0.989 & & \\
\hline & & 5 & 1.686 & 0.548 & 0.033 & 0.060 & 0.124 & 0.079 & 0.65 & 0.979 & & \\
\hline
\end{tabular}


Table 2. (Continued).

\begin{tabular}{|c|c|c|c|c|c|c|c|c|c|c|c|}
\hline $\begin{array}{l}\text { Core-Section } \\
\text { (level in } \mathrm{cm} \text { ) }\end{array}$ & Grain & $\mathrm{Fe}$ & $\mathrm{Ti}$ & $\mathrm{Mn}$ & $\mathrm{Si}$ & Al & $\mathrm{Mg}$ & $x$ & $z$ & $\bar{x}$ & $\bar{z}$ \\
\hline \multicolumn{12}{|l|}{ Hole 524 (Cont.) } \\
\hline $30-2,110$ & $\begin{array}{l}1 \\
2 \\
3 \\
4 \\
5\end{array}$ & $\begin{array}{l}1.683 \\
1.774 \\
1.771 \\
1.629 \\
1.700\end{array}$ & $\begin{array}{l}0.576 \\
0.539 \\
0.562 \\
0.522 \\
0.543\end{array}$ & $\begin{array}{l}0.042 \\
0.059 \\
0.043 \\
0.068 \\
0.056\end{array}$ & $\begin{array}{l}0.027 \\
0.041 \\
0.051 \\
0.062 \\
0.041\end{array}$ & $\begin{array}{l}0.110 \\
0.078 \\
0.089 \\
0.056 \\
0.113\end{array}$ & $\begin{array}{l}0.064 \\
0.029 \\
0.045 \\
0.062 \\
0.074\end{array}$ & $\begin{array}{l}0.69 \\
0.64 \\
0.66 \\
0.65 \\
0.65\end{array}$ & $\begin{array}{l}1.02 \\
0.99 \\
0.88 \\
1.34 \\
1.00\end{array}$ & $0.66(0.02)$ & $1.05(0.15)$ \\
\hline $31-2,69$ & $\begin{array}{l}1 \\
2 \\
3 \\
4 \\
5 \\
6\end{array}$ & $\begin{array}{l}1.989 \\
2.003 \\
2.024 \\
2.032 \\
2.058 \\
2.043\end{array}$ & $\begin{array}{l}0.628 \\
0.595 \\
0.603 \\
0.595 \\
0.594 \\
0.570\end{array}$ & $\begin{array}{l}0.025 \\
0.027 \\
0.027 \\
0.031 \\
0.029 \\
0.023\end{array}$ & $\begin{array}{l}0.008 \\
0.006 \\
0.006 \\
0.006 \\
0.006 \\
0.008\end{array}$ & $\begin{array}{l}0.025 \\
0.056 \\
0.033 \\
0.025 \\
0.023 \\
0.031\end{array}$ & $\begin{array}{l}0.040 \\
0.052 \\
0.029 \\
0.040 \\
0.036 \\
0.046\end{array}$ & $\begin{array}{l}0.69 \\
0.65 \\
0.66 \\
0.65 \\
0.65 \\
0.63\end{array}$ & $\begin{array}{l}0.52 \\
0.49 \\
0.51 \\
0.50 \\
0.47 \\
0.53\end{array}$ & 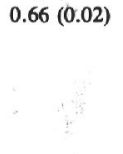 & $0.50(0.02)$ \\
\hline $33-2,52$ & $\begin{array}{l}1 \\
2 \\
3 \\
4 \\
5 \\
6 \\
7\end{array}$ & $\begin{array}{l}1.857 \\
1.915 \\
1.915 \\
1.956 \\
1.798 \\
1.995 \\
1.991\end{array}$ & $\begin{array}{l}0.556 \\
0.543 \\
0.591 \\
0.543 \\
0.525 \\
0.611 \\
0.596\end{array}$ & $\begin{array}{l}0.026 \\
0.021 \\
0.020 \\
0.027 \\
0.018 \\
0.021 \\
0.023\end{array}$ & $\begin{array}{l}0.022 \\
0.012 \\
0.008 \\
0.023 \\
0.008 \\
0.008 \\
0.010\end{array}$ & $\begin{array}{l}0.122 \\
0.159 \\
0.100 \\
0.146 \\
0.128 \\
0.082 \\
0.077\end{array}$ & $\begin{array}{l}0.136 \\
0.149 \\
0.043 \\
0.140 \\
0.132 \\
0.044 \\
0.065\end{array}$ & $\begin{array}{l}0.61 \\
0.58 \\
0.66 \\
0.57 \\
0.60 \\
0.66 \\
0.65\end{array}$ & $\begin{array}{l}0.58 \\
0.41 \\
0.61 \\
0.33 \\
0.84 \\
0.44 \\
0.44\end{array}$ & $0.62(0.04)$ & $0.52(0.16)$ \\
\hline $33-2,98$ & $\begin{array}{l}1 \\
2 \\
3 \\
4 \\
5\end{array}$ & $\begin{array}{l}2.097 \\
2.061 \\
2.126 \\
1.994 \\
2.092\end{array}$ & $\begin{array}{l}0.617 \\
0.623 \\
0.616 \\
0.569 \\
0.563\end{array}$ & $\begin{array}{l}0.017 \\
0.023 \\
0.024 \\
0.021 \\
0.028\end{array}$ & $\begin{array}{l}0.006 \\
0.008 \\
0.006 \\
0.006 \\
0.021\end{array}$ & $\begin{array}{l}0.060 \\
0.051 \\
0.067 \\
0.045 \\
0.085\end{array}$ & $\begin{array}{l}0.030 \\
0.030 \\
0.017 \\
0.016 \\
0.040\end{array}$ & $\begin{array}{l}0.65 \\
0.67 \\
0.65 \\
0.64 \\
0.60\end{array}$ & $\begin{array}{l}0.31 \\
0.36 \\
0.25 \\
0.66 \\
0.31\end{array}$ & $0.64(0.02)$ & $0.38(0.15)$ \\
\hline $33-2,29$ & $\begin{array}{l}1 \\
2 \\
3 \\
4 \\
5 \\
6\end{array}$ & $\begin{array}{l}2.165 \\
2.137 \\
2.139 \\
2.170 \\
2.133 \\
2.180\end{array}$ & $\begin{array}{l}0.529 \\
0.570 \\
0.581 \\
0.567 \\
0.554 \\
0.574\end{array}$ & $\begin{array}{l}0.020 \\
0.022 \\
0.022 \\
0.022 \\
0.022 \\
0.022\end{array}$ & $\begin{array}{l}0.039 \\
0.044 \\
0.059 \\
0.035 \\
0.057 \\
0.069\end{array}$ & $\begin{array}{l}0.146 \\
0.122 \\
0.112 \\
0.139 \\
0.122 \\
0.138\end{array}$ & $\begin{array}{l}0.094 \\
0.083 \\
0.088 \\
0.084 \\
0.098 \\
0.089\end{array}$ & $\begin{array}{l}0.53 \\
0.57 \\
0.58 \\
0.56 \\
0.56 \\
0.56\end{array}$ & $\begin{array}{r}0.011 \\
0.038 \\
0.000 \\
-0.033 \\
0.028 \\
-0.132\end{array}$ & $0.56(0.02)$ & $-0.02(0.06)$ \\
\hline $33-3,100$ & $\begin{array}{l}1 \\
1 \\
2 \\
2 \\
3 \\
3\end{array}$ & $\begin{array}{l}2.175 \\
2.143 \\
2.176 \\
2.123 \\
2.168 \\
2.135\end{array}$ & $\begin{array}{l}0.627 \\
0.611 \\
0.596 \\
0.611 \\
0.573 \\
0.585\end{array}$ & $\begin{array}{l}0.024 \\
0.022 \\
0.020 \\
0.022 \\
0.022 \\
0.020\end{array}$ & $\begin{array}{l}0.004 \\
0.007 \\
0.007 \\
0.006 \\
0.007 \\
0.004\end{array}$ & $\begin{array}{l}0.098 \\
0.105 \\
0.117 \\
0.098 \\
0.130 \\
0.118\end{array}$ & $\begin{array}{l}0.107 \\
0.105 \\
0.117 \\
0.092 \\
0.117 \\
0.105\end{array}$ & $\begin{array}{l}0.62 \\
0.61 \\
0.59 \\
0.62 \\
0.57 \\
0.59\end{array}$ & $\begin{array}{r}-0.06 \\
0.01 \\
-0.06 \\
0.09 \\
-0.03 \\
0.06\end{array}$ & $0.60(0.02)$ & $0.00(0.06)$ \\
\hline $33-33,131$ & $\begin{array}{l}1 \\
2 \\
3 \\
4 \\
5\end{array}$ & $\begin{array}{l}2.103 \\
1.938 \\
2.116 \\
2.122 \\
2.041\end{array}$ & $\begin{array}{l}0.626 \\
0.565 \\
0.613 \\
0.598 \\
0.615\end{array}$ & $\begin{array}{l}0.021 \\
0.027 \\
0.022 \\
0.024 \\
0.032\end{array}$ & $\begin{array}{l}0.006 \\
0.020 \\
0.004 \\
0.009 \\
0.017\end{array}$ & $\begin{array}{l}0.069 \\
0.076 \\
0.047 \\
0.076 \\
0.083\end{array}$ & $\begin{array}{l}0.087 \\
0.076 \\
0.069 \\
0.080 \\
0.068\end{array}$ & $\begin{array}{l}0.65 \\
0.63 \\
0.64 \\
0.62 \\
0.65\end{array}$ & $\begin{array}{l}0.16 \\
0.58 \\
0.23 \\
0.16 \\
0.26\end{array}$ & $0.64(0.01)$ & $0.28(0.16)$ \\
\hline
\end{tabular}

Note: The metal analyses are presented as the number of cations per 4 oxygen ions, the oxygen determined by difference. $x$ is the number of four-valent ions in the equivalent stoichiometric titanomagnetite. $z$ is the oxidation parameter derived as described in the text. Numbers in parentheses are standard deviations.

the trend definitely. For non-interacting monodomain grains with randomly oriented easy axes (uniaxial symmetry), the model $N R M / M_{R S}$ ratio (NRM being a thermoremanence) is as follows (Néel, 1949):

$$
\frac{N R M}{M_{R S}}=\frac{2 \nu M_{S}^{0}\left(T_{B}\right) H}{3 \mathrm{k} T_{B}}
$$

where $\nu$ is the volume of the grain, $T_{B}$ is the blocking temperature, $H$ is the field in which the $N R M$ was acquired (the NRM is assumed not to have decayed due to realignment of grain magnetization), and the zero superscript refers to the spontaneous magnetization before alteration (which is assumed to be uniform throughout the grain). Maghemitization will generally not change the $N R M / M_{R S}$ ratio (Ozdemir and O'Reilly, 1982), so an increase in this ratio may indicate an increase in grain volume. $T_{B}$ is also a function of grain volume. The "hardness" of $M_{R S}$ as measured by the $M D F$ decreases with increasing depth in the flow. Both increasing alteration (reducing magnetic anisotropy) and increasing grain volume could account for such a trend.

\section{Hysteresis Parameters and Initial Susceptibility}

Magnetic hysteresis loops were obtained by using a vibrating sample magnetometer, and the parameters of the loops, saturation magnetization $M_{s}$, saturation remanence $M_{R S}$, and coercive force $H_{c}$ are listed in Table 3. The slope of the high-field section of the loops, the highfield susceptibility $\chi_{p}$, and the low-field susceptibility $\chi_{0}$ determined by using a susceptibility bridge are also listed.

The saturation magnetization rises systematically with increasing depth in the flow. In determining $M_{s}$, the effect of high-field susceptibility $\chi_{p}$ has been removed. $\boldsymbol{M}_{s}$ therefore reflects the concentration and composition of the magnetic phases. If we assume that alteration will generally reduce $M_{s}$ at room temperature, the rise observed in the flow must indicate an increase in the concentration of the mineral. For superparamagnetic grains of titanomagnetite with a spontaneous magnetization of $1.1 \times 10^{5} \mathrm{~A} / \mathrm{m}$, only grains with diameter less than about $50 \AA$ would fail to achieve more than $50 \%$ of the saturation moment at room temperature in the available field, $8 \times 10^{5} \mathrm{~A} / \mathrm{m}$. 
Table 3. Summary of magnetic properties.

\begin{tabular}{|c|c|c|c|c|c|c|c|c|c|c|c|}
\hline $\begin{array}{l}\text { Core-Section } \\
\text { (level in } \mathrm{cm})\end{array}$ & $\begin{array}{c}N R M \\
\left(\mathrm{Am}^{2} / \mathrm{kg}\right)\end{array}$ & $\begin{array}{c}M_{R S} \\
\left(\mathrm{Am}^{2} / \mathrm{kg}\right)\end{array}$ & $N R M / M_{R S}$ & $\begin{array}{l}M D F \\
(\mathrm{~A} / \mathrm{m})\end{array}$ & $\begin{array}{c}M_{S} \\
\left(\mathrm{Am}^{2} / \mathrm{kg}\right)\end{array}$ & $\begin{array}{c}H_{c} \\
(\mathrm{~A} / \mathrm{m})\end{array}$ & $M_{R S} / M_{S}$ & $\begin{array}{c}\underset{x}{p} p \\
\left(\mathrm{~m}^{3} / \mathrm{kg}\right)\end{array}$ & $\left(\mathrm{m}^{3} / \mathrm{kg}\right)$ & $Q$ & $\begin{array}{l}\text { Basalt } \\
\text { type }\end{array}$ \\
\hline \multicolumn{12}{|l|}{ Hole 519A } \\
\hline $\begin{array}{l}9-1,11 \\
9-1,27 \\
9-1,80 \\
9-2,29 \\
9-3,145 \\
9-4,40 \\
9-4,84\end{array}$ & $\begin{array}{l}2.77 \times 10^{-4} \\
4.52 \times 10^{-4} \\
4.90 \times 10^{-4} \\
1.98 \times 10^{-4} \\
6.33 \times 10^{-4} \\
6.30 \times 10^{-4} \\
4.43 \times 10^{-4}\end{array}$ & $\begin{array}{l}9.17 \times 10^{-2} \\
1.14 \times 10^{-1} \\
1.08 \times 10^{-1} \\
1.16 \times 10^{-1} \\
1.26 \times 10^{-1} \\
1.00 \times 10^{-1} \\
6.25 \times 10^{-2}\end{array}$ & $\begin{array}{l}3.95 \times 10^{-3} \\
3.01 \times 10^{-3} \\
4.51 \times 10^{-3} \\
1.72 \times 10^{-3} \\
5.00 \times 10^{-3} \\
6.30 \times 10^{-3} \\
7.09 \times 10^{-3}\end{array}$ & $\begin{array}{r}2.23 \times 10^{4} \\
1.59 \times 10^{4} \\
9.2 \times 10^{3} \\
6.4 \times 10^{3} \\
8.0 \times 10^{3} \\
7.2 \times 10^{3} \\
3.6 \times 10^{3}\end{array}$ & $\begin{array}{l}1.95 \times 10^{-1} \\
1.93 \times 10^{-1} \\
2.56 \times 10^{-1} \\
3.00 \times 10^{-1} \\
2.94 \times 10^{-1} \\
3.93 \times 10^{-1} \\
4.13 \times 10^{-1}\end{array}$ & $\begin{array}{l}1.95 \times 10^{4} \\
2.00 \times 10^{4} \\
1.19 \times 10^{4} \\
1.10 \times 10^{4} \\
1.08 \times 10^{4} \\
7.31 \times 10^{3} \\
4.29 \times 10^{3}\end{array}$ & $\begin{array}{l}0.47 \\
0.59 \\
0.42 \\
0.38 \\
0.43 \\
0.25 \\
0.15\end{array}$ & $\begin{array}{c}- \\
- \\
2.94 \times 10^{-7} \\
2.52 \times 10^{-7} \\
3.16 \times 10^{-7} \\
3.00 \times 10^{-7} \\
2.75 \times 10^{-7}\end{array}$ & $\begin{array}{l}1.82 \times 10^{-6} \\
1.09 \times 10^{-6} \\
1.30 \times 10^{-6} \\
1.32 \times 10^{-6} \\
1.44 \times 10^{-6} \\
3.50 \times 10^{-6} \\
4.61 \times 10^{-6}\end{array}$ & $\begin{array}{c}7.1 \\
19 \\
18 \\
7.0 \\
20 \\
8.4 \\
4.5\end{array}$ & Flow \\
\hline \multicolumn{12}{|l|}{ Hole 522B } \\
\hline $\begin{array}{l}3-4,61 \\
4-1,17 \\
4-1,43 \\
5-2,30 \\
5-2,75 \\
5-2,139 \\
5-3,41\end{array}$ & $\begin{array}{l}1.28 \times 10^{-3} \\
1.21 \times 10^{-3} \\
1.00 \times 10^{-3} \\
5.80 \times 10^{-4} \\
3.28 \times 10^{-3} \\
2.12 \times 10^{-3} \\
3.55 \times 10^{-3}\end{array}$ & $\begin{array}{r}1.15 \times 10^{-1} \\
1.21 \times 10^{-1} \\
1.00 \times 10^{-1} \\
6.6 \times 10^{-2} \\
1.54 \times 10^{-1} \\
1.21 \times 10^{-1} \\
1.21 \times 10^{-1}\end{array}$ & $\begin{array}{r}1.11 \times 10^{-2} \\
0.91 \times 10^{-2} \\
1.94 \times 10^{-2} \\
8.8 \times 10^{-3} \\
2.13 \times 10^{-2} \\
1.75 \times 10^{-2} \\
2.93 \times 10^{-2}\end{array}$ & $\begin{array}{l}1.03 \times 10^{4} \\
3.82 \times 10^{3} \\
3.26 \times 10^{3} \\
2.24 \times 10^{4} \\
5.97 \times 10^{3} \\
3.34 \times 10^{3} \\
5.33 \times 10^{3}\end{array}$ & $\begin{array}{l}2.18 \times 10^{-1} \\
5.93 \times 10^{-1} \\
5.29 \times 10^{-1} \\
1.04 \times 10^{-2} \\
5.24 \times 10^{-1} \\
5.54 \times 10^{-1} \\
5.03 \times 10^{-1}\end{array}$ & $\begin{array}{r}1.54 \times 10^{4} \\
5.8 \times 10^{3} \\
4.1 \times 10^{3} \\
2.78 \times 10^{4} \\
8.5 \times 10^{3} \\
7.4 \times 10^{3} \\
5.4 \times 10^{3}\end{array}$ & $\begin{array}{l}0.48 \\
0.20 \\
0.20 \\
0.61 \\
0.29 \\
0.21 \\
0.26\end{array}$ & $\begin{array}{l}1.49 \times 10^{-7} \\
1.75 \times 10^{-7} \\
2.01 \times 10^{-7} \\
1.48 \times 10^{-7} \\
1.82 \times 10^{-7} \\
1.86 \times 10^{-7} \\
1.67 \times 10^{-7}\end{array}$ & $\begin{array}{l}1.66 \times 10^{-6} \\
5.55 \times 10^{-6} \\
6.37 \times 10^{-6} \\
9.85 \times 10^{-7} \\
3.41 \times 10^{-6} \\
5.48 \times 10^{-6} \\
4.59 \times 10^{-6}\end{array}$ & $\left.\begin{array}{c}36 \\
6.9 \\
14 \\
28 \\
45 \\
18 \\
36\end{array}\right)$ & $\begin{array}{l}\text { Pillow } \\
\text { Flow }\end{array}$ \\
\hline \multicolumn{12}{|l|}{ Hole 524} \\
\hline $\begin{array}{l}29-2,28 \\
30-1,141 \\
30-2,52 \\
30-2,110 \\
31-2,69 \\
31-2,79 \\
33-2,52 \\
33-2,98 \\
33-3,29 \\
33-3,100 \\
33-3,105 \\
33-3,127 \\
33-3,131\end{array}$ & $\begin{array}{l}1.24 \times 10^{-3} \\
1.45 \times 10^{-3} \\
2.13 \times 10^{-3} \\
2.50 \times 10^{-3} \\
5.15 \times 10^{-4} \\
3.97 \times 10^{-3} \\
2.46 \times 10^{-3} \\
1.40 \times 10^{-3} \\
5.06 \times 10^{-4} \\
7.10 \times 10^{-4} \\
1.71 \times 10^{-3} \\
5.72 \times 10^{-3} \\
9.46 \times 10^{-4}\end{array}$ & $\begin{array}{l}1.47 \times 10^{-1} \\
1.72 \times 10^{-1} \\
2.21 \times 10^{-1} \\
2.31 \times 10^{-1} \\
1.24 \times 10^{-4} \\
1.20 \times 10^{-1} \\
1.75 \times 10^{-1} \\
1.76 \times 10^{-1} \\
1.35 \times 10^{-1} \\
1.12 \times 10^{-1} \\
1.10 \times 10^{-1} \\
1.20 \times 10^{-1} \\
1.24 \times 10^{-1}\end{array}$ & $\begin{array}{l}0.84 \times 10^{-2} \\
0.84 \times 10^{-2} \\
0.96 \times 10^{-2} \\
1.08 \times 10^{-2} \\
1.24 \times 10^{-1} \\
6.72 \times 10^{-2} \\
1.41 \times 10^{-2} \\
0.79 \times 10^{-2} \\
0.37 \times 10^{-2} \\
0.63 \times 10^{-2} \\
2.73 \times 10^{-2} \\
10.9 \times 10^{-2} \\
0.76 \times 10^{-2}\end{array}$ & $\begin{array}{c}2.15 \times 10^{3} \\
2.07 \times 10^{3} \\
1.52 \times 10^{3} \\
1.83 \times 10^{3} \\
3.58 \times 10^{3} \\
- \\
1.83 \times 10^{3} \\
1.51 \times 10^{3} \\
3.34 \times 10^{3} \\
4.69 \times 10^{3} \\
- \\
- \\
1.75 \times 10^{3}\end{array}$ & $\begin{array}{r}12.7 \times 10^{-1} \\
10.6 \times 10^{-1} \\
13.5 \times 10^{-1} \\
14.8 \times 10^{-1} \\
13.2 \times 10^{-1} \\
12.6 \times 10^{-1} \\
9.8 \times 10^{-1} \\
12.2 \times 10^{-1} \\
12.5 \times 10^{-1} \\
12.2 \times 10^{-1} \\
12.4 \times 10^{-1} \\
12.3 \times 10^{-1} \\
12.1 \times 10^{-1}\end{array}$ & $\begin{array}{l}3.3 \times 10^{3} \\
3.4 \times 10^{3} \\
5.6 \times 10^{3} \\
5.4 \times 10^{3} \\
2.6 \times 10^{3} \\
2.6 \times 10^{3} \\
5.1 \times 10^{3} \\
3.4 \times 10^{3} \\
2.9 \times 10^{3} \\
2.5 \times 10^{3} \\
2.5 \times 10^{3} \\
2.7 \times 10^{3} \\
2.8 \times 10^{3}\end{array}$ & $\begin{array}{l}0.12 \\
0.16 \\
0.16 \\
0.15 \\
0.10 \\
0.09 \\
0.18 \\
0.14 \\
0.10 \\
0.09 \\
0.09 \\
0.10 \\
0.11\end{array}$ & $\begin{array}{l}2.08 \times 10^{-7} \\
1.85 \times 10^{-7} \\
2.44 \times 10^{-7} \\
2.60 \times 10^{-7} \\
2.01 \times 10^{-7} \\
2.03 \times 10^{-7} \\
2.04 \times 10^{-7} \\
2.07 \times 10^{-7} \\
2.16 \times 10^{-7} \\
2.08 \times 10^{-7} \\
2.14 \times 10^{-7} \\
2.41 \times 10^{-7} \\
2.09 \times 10^{-7}\end{array}$ & $\begin{array}{r}1.51 \times 10^{-5} \\
1.20 \times 10^{-5} \\
1.59 \times 10^{-5} \\
1.47 \times 10^{-5} \\
1.54 \times 10^{-5} \\
1.64 \times 10^{-5} \\
9.0 \times 10^{-6} \\
1.31 \times 10^{-5} \\
1.33 \times 10^{-5} \\
1.63 \times 10^{-5} \\
1.67 \times 10^{-5} \\
1.27 \times 10^{-5} \\
1.13 \times 10^{-5}\end{array}$ & $\begin{array}{c}.3 .8 \\
5.7 \\
6.2 \\
7.9 \\
1.6 \\
11 \\
13 \\
4.9 \\
1.8 \\
2.0 \\
4.7 \\
20 \\
3.8\end{array}$ & $\begin{array}{c}\text { Lower sill, } \\
\text { central } \\
\text { part }\end{array}$ \\
\hline
\end{tabular}

Note: Symbols are defined in text.

The coercive force falls systematically with increasing depth in the flow. The reduction can be accounted for by decreasing magnetic anisotropy and/or increasing grain volume. The values of the ratio $M_{R S} / M_{s}$ also tend to fall with increasing depth, although the value is approximately constant and indicates the presence of stable monodomain or pseudo-single-domain material in most of the flow (Day, 1977). The initial susceptibility, which depends on the concentration of magnetic material and magnetic "hardness," increases with increasing depth in the flow, consistent with the variations in $M D F$ and $H_{c}$. The Koenigsberger ratio $Q$ (which is equivalent to $N R M / \chi_{0} H ; H$ for this latitude equals $21.5 \mathrm{~A} / \mathrm{m}$ ) shows no systematic variation with depth, the range of values suggesting the monodomain/pseudo-singledomain state. The fall in $H_{c}$ and $M_{R S} / M_{s}$ with increasing depth could also be explained, in principle, by an increasing superparamagnetic fraction (i.e., decreasing grain volume). However, this explanation seems improbable in view of the small volume of material likely to exist in the superparamagnetic size range $(0-500 \AA)$ and the optical evidence to the contrary. Furthermore, there is no other magnetic evidence for a significant superparamagnetic fraction, in the form of high susceptibility $-500 \AA$ grains would achieve $50 \%$ of saturation in a field of only $120 \mathrm{~A} / \mathrm{m}$-or "pinched" hysteresis loops.

\section{Thermomagnetic Analysis}

Samples of whole rock (around $0.15 \mathrm{~g}$ of powder and chips) were evacuated overnight in quartz ampules and sealed off. The variation of induced high-field magnetic moment with temperature running from room tempera- ture to about $700^{\circ} \mathrm{C}$ and returning to room temperature (heating/cooling rate $\approx 2.5^{\circ} / \mathrm{min}$.) was determined by using a magnetic translation balance and an applied field of $6.96 \times 10^{5} \mathrm{~A} / \mathrm{m}(8.75 \mathrm{kOe})$. The heating and cooling runs are in general different, indicating that some physico-chemical changes took place during heating. Such behavior is common in submarine basalts and is generally believed to be due to the presence of structurally metastable titanomaghemites. Various types of behavior are observed, and the thermomagnetic curves may be assigned to a number of categories (e.g., Wasilewski, 1968). Here we adopt a simple classification illustrated (with samples from Holes 522B and 524) in Figure 1. Curves of Type A (Fig. 1A) show an uninterrupted fall in magnetization with ascending temperature to a Curie point below about $300^{\circ} \mathrm{C}$. Magnetization decreases gradually above this temperature, consistent with Curie law paramagnetic behavior, although there is a slight rise and fall between 300 and $400^{\circ} \mathrm{C}$. The cooling curve is slightly displaced from the heating curve. As a working hypothesis we might suppose that a Type A curve corresponds to a slightly maghemitized titanomagnetite. In curves of Types B and C (Figs. 1B and 1C), the heating and cooling curves are quite different. Each heating curve shows an initial fall in magnetization that is interrupted at 250 to $300^{\circ} \mathrm{C}$ by a rise as temperature increases further. The rise may be smooth or have steps, depending on the heating regime; the exact form of the curve depends on the interplay of the rate at which inversion takes place at a given temperature, the rate at which temperature rises, and the intrinsic fall in magnetization of the inversion product with rising tem- 
A

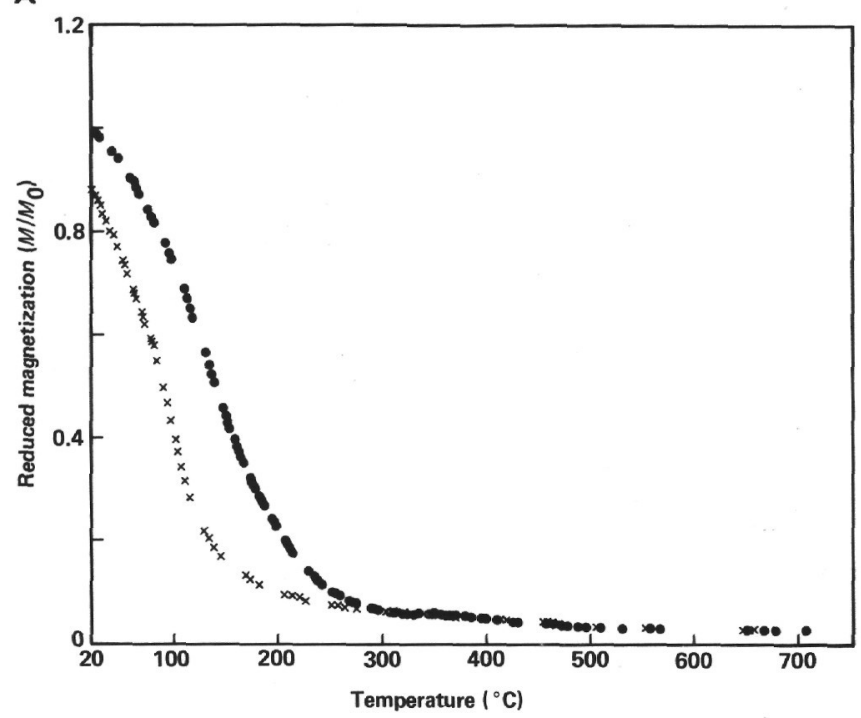

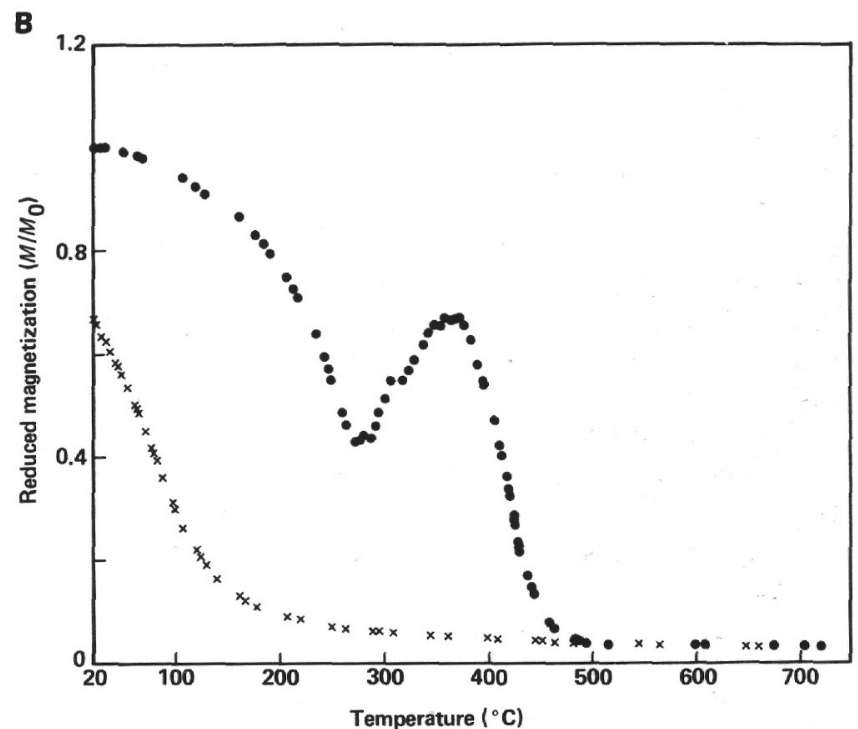

C

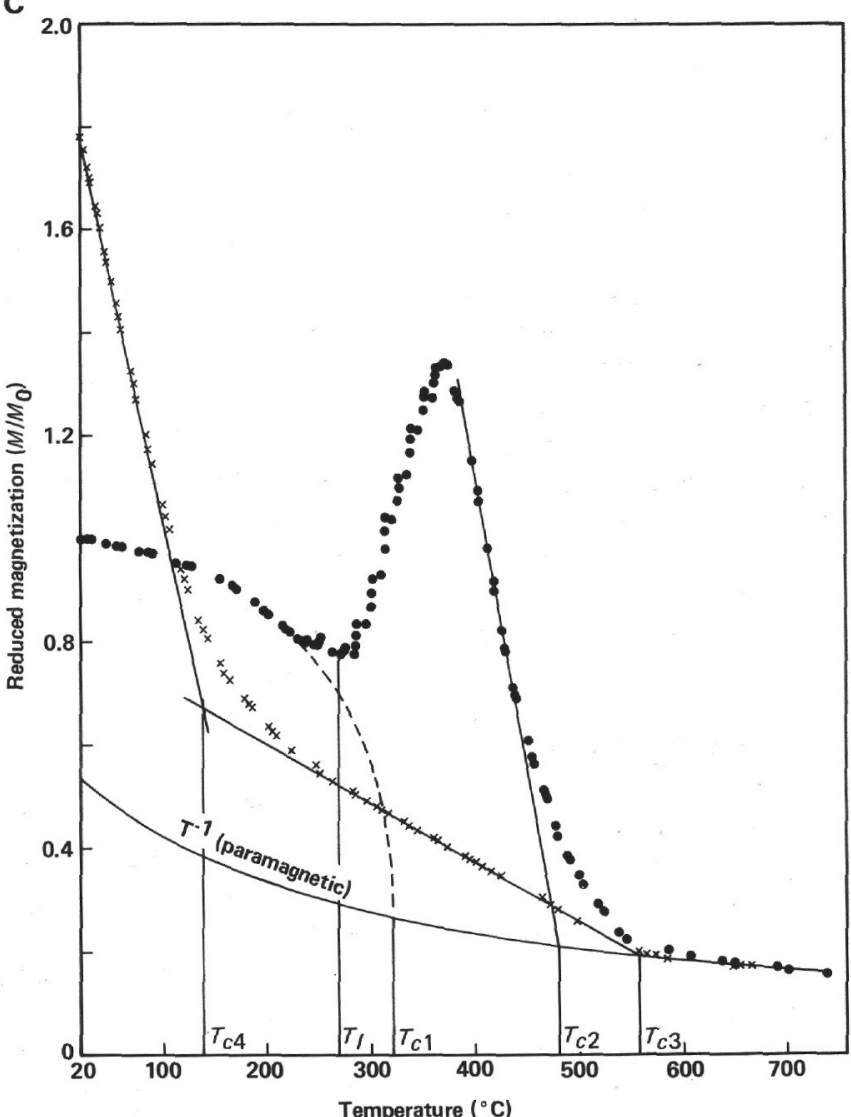

- Heating

$x$ Cooling

Figure 1. High-field thermomagnetic curves. A. Curve is designated Type A and is believed to correspond to a titanomaghemite with only a slight degree of maghemitization. Sample 524-33-3, $100 \mathrm{~cm}$. B. Curve is designated Type B and is believed to correspond to a moderate degree of maghemitization. Sample 524-30-1, $141 \mathrm{~cm}$. C. Curve is designated Type $\mathrm{C}$ and is believed to correspond to a high degree of maghemitization. Curve also defines $T_{c 1}, T_{c 2}$, etc. and the Curie law $\left(T^{-1}\right)$ extrapolation of the induced magnetization from above the highest Curie point. Sample $522 \mathrm{~B}-5-2,30 \mathrm{~cm}$.

perature. However, after a temperature of about $350^{\circ} \mathrm{C}$ is reached, magnetization decreases without further interruption to a Curie temperature in the range between 400 and $500^{\circ} \mathrm{C}$. The cooling curve rises steadily, more rapidly in the lower temperature section. In curves of Type B, the evidence for the inversion process (the peak in the heating curve), although pronounced, is not as dramatic as in curves of Type $C$. The ratio of high-fieldinduced magnetization after and before the run $\left(M_{F} /\right.$ $M_{I}$ ) is also different in the two categories. (For $M_{F} / M_{I}$ ratios for synthetic titanomaghemites see Özdemir and O'Reilly, 1981.) It is the high-field magnetization due to 
the magnetic fraction that is of interest. The contribution from iron-bearing paramagnetic minerals must be determined and allowed for; it may be calculated by using values of $\chi_{p}$ from the hysteresis loops. The contribution thus found can be compared with the paramagnetism observed above the Curie temperatures. If the differences in temperature are allowed for (which may be done by using the Curie law), agreement is good in some cases. Typically, curves of Types A and B have $M_{F} / M_{I}$ ratios near unity $(\sim 0.8-1.0)$, and curves of Type $C$ have ratios that are significantly higher $(\geq 2)$. We will suppose that basalt that produces a Type $C$ curve is characterized by a higher degree of maghemitization than a basalt that produces a Type B curve. The fact that the cooling curve does not trace back the upper section of the heating curve suggests that inversion has not gone to completion in the time available and/or that some other process takes place at higher temperature. The shape of the cooling curve is of interest. It may be that in the course of inversion the grains are subdivided by the nonmagnetic, possibly Ti-rich, inversion product, leaving the magnetic product in a finely divided state. The presence of inclusions, shrinkage cracks, and other elements of microstructure may also influence the course of inversion and the effective grain size of the inversion product. The Curie point of this (possibly) superparamagnetic phase may then be difficult to distinguish in the available magnetic field.

These complicated curves may be characterized in a simplified way by the following critical or characteristic temperatures. $T_{c 1}$ is the first Curie temperature encountered on the heating run. This is the Curie point of the uninverted titanomaghemite. $T_{c 2}$ is the last significant temperature observed on the heating run and is the $\mathrm{Cu}-$ rie point of the first magnetic inversion product formed. The onset of inversion will be lower than or near the trough in the heating run. The trough in the heating run is designated $T_{I}$ and is taken as being an upper limit for the onset of inversion. When $T_{I}<T_{c 1}, T_{c 1}$ must be determined by extrapolation, and therefore carry a correspondingly larger uncertainty. $T_{c 3}$ and $T_{c 4}$ characterize the cooling curve; they are the intercepts of the higher and lower sections of this curve on the effective zero magnetization/temperature axis (see Fig. 1C).

Table 4 summarizes the results of the thermomagnetic analysis of Hole 519A. The parameters vary systematically with increasing depth. $T_{c 1}$ decreases, the result of either a decrease in $\mathrm{Fe}^{3+} / \mathrm{Fe}^{2+}$ ratio or an increase in the concentration of nonmagnetic ions (i.e., an increase in $x$ or a decrease in $z$ ) as depth increases. $T_{c 2}$ also decreases with increasing depth, although $T_{c 3}$ is approximately constant. It may be that as depth increases the firstformed inversion product contains more titanium or other nonmagnetic ions which in the final phase of inversion, at high temperature, migrate out, leaving a higher Curie point phase. Of course, it must be remembered that the precisely defined critical points are a feature of theoretical models developed for ordering phenomena and that real materials do not necessarily exhibit sharply definable transitions. Experimentally determined Curie points therefore rely on the adoption of a practical defi-
Table 4. Results (in ${ }^{\circ} \mathrm{C}$ ) of thermomagnetic analysis.

\begin{tabular}{|c|c|c|c|c|c|c|c|}
\hline $\begin{array}{l}\text { Core-Section } \\
\text { (level in } \mathrm{cm})\end{array}$ & $T_{c 1}$ & $T_{\mathrm{c} 2}$ & $T_{c 3}$ & $T_{c 4}$ & $T_{I}$ & $M_{F} / M_{I}$ & $\begin{array}{c}\text { Curve } \\
\text { type }\end{array}$ \\
\hline \multicolumn{8}{|l|}{ Hole 519A } \\
\hline $\begin{array}{l}9-1,11 \\
9-1,27 \\
9-2,29 \\
9-3,145 \\
9-4,40 \\
9-4,84\end{array}$ & $\begin{array}{l}330 \pm 20 \\
264 \pm 25 \\
285 \pm 8 \\
239 \pm 2 \\
200 \pm 5 \\
201 \pm 2\end{array}$ & $\begin{array}{l}515 \pm 5 \\
506 \pm 4 \\
507 \pm 5 \\
498 \pm 3 \\
-37 \\
437 \pm 15\end{array}$ & $\begin{array}{c}559 \pm 6 \\
524 \pm 12 \\
546 \pm 13 \\
545 \pm 25 \\
= \\
=\end{array}$ & $\begin{array}{l}267 \pm 2 \\
172 \pm 3 \\
180 \pm 14 \\
173 \pm 5 \\
137 \pm 4 \\
137 \pm 4\end{array}$ & $\begin{array}{c}306 \pm 8 \\
291 \pm 2 \\
305 \pm 5 \\
290 \pm 4 \\
- \\
-\end{array}$ & $\begin{array}{l}2.17 \\
1.39 \\
1.95 \\
1.49 \\
0.94 \\
0.82\end{array}$ & $\begin{array}{l}\text { C } \\
C \text { (B) } \\
\text { C } \\
\text { C (B) } \\
\text { A (B) } \\
\text { A }\end{array}$ \\
\hline \multicolumn{8}{|l|}{ Hole 522B } \\
\hline $\begin{array}{l}3-4,61 \\
4-1,11^{\mathrm{b}} \\
4-1,43^{\mathrm{b}} \\
4-1,108^{\mathrm{b}} \\
5-2,30 \\
5-2,75 \\
5-2,102 \\
5-2,139^{\mathrm{b}} \\
5-3,41^{\mathrm{b}}\end{array}$ & $\begin{array}{l}295 \pm 20 \\
170 \\
170 \\
240,330 \\
320 \pm 30 \\
221 \pm 2 \\
193 \pm 4 \\
160 \\
210\end{array}$ & $\begin{array}{l}479 \pm 3 \\
407 \pm 4 \\
556 \pm 7\end{array}$ & $\begin{array}{c}557 \pm 12 \\
556 \pm 7\end{array}$ & $\begin{array}{l}140 \pm 5 \\
142 \pm 4 \\
144 \pm 4\end{array}$ & $\begin{array}{l}269 \pm 5 \\
297 \pm 5 \\
317 \pm 5\end{array}$ & $\begin{array}{l}2.72 \\
0.73 \\
0.82\end{array}$ & $\begin{array}{l}\text { C } \\
\text { A (B) } \\
\text { A (B) }\end{array}$ \\
\hline \multicolumn{8}{|l|}{ Hole 524} \\
\hline $\begin{array}{l}29-2,28 \\
30-1,141 \\
30-2,110^{\mathrm{b}} \\
31-2,79^{\mathrm{b}} \\
33-1,101^{\mathrm{b}} \\
33-2,52 \\
33-3,29^{\mathrm{b}} \\
33-3,100 \\
33-3,105^{\mathrm{b}} \\
33-3,127^{\mathrm{b}} \\
33-3,131^{\mathrm{b}}\end{array}$ & $\begin{array}{l}28 \pm 10 \\
292 \pm 25 \\
350 \\
360 \\
350 \\
297 \pm 15 \\
300 \\
214 \pm 2 \\
260 \\
260 \\
250\end{array}$ & $\begin{array}{l}461 \pm 3 \\
422 \pm 10\end{array}$ & $\begin{array}{c}492 \pm 8 \\
-\end{array}$ & $\begin{array}{l}194 \pm .4 \\
141 \pm 3\end{array}$ & $\begin{array}{l}280 \pm 3 \\
320 \pm 5\end{array}$ & $\begin{array}{l}0.80 \\
0.88\end{array}$ & $\begin{array}{l}\text { B (C) } \\
\text { A }\end{array}$ \\
\hline
\end{tabular}

Note: Symbols are defined in text and Fig. 1C.

Curve shows a tendency toward the type in parentheses.
bample was analyzed in a different set of experiments, and $T_{c 2}$ etc. were not determined.

nition of $T_{c}$ or a conventional procedure for determining $T_{c}$ that may have a subjective element. $T_{c 4}$ is not necessarily a Curie point; if the inverted material does exhibit superparamagnetism, it is affected by the effective grain size distribution, the temperature dependence of magnetization and (presumably shape) anisotropy, and the applied field. The systematic reduction in $T_{c 4}$ with increasing depth may correspond to a reduction in effective grain size (which may reflect the pre-inversion grain size) or a reduction in intrinsic magnetization. The onset of inversion, $T_{i}$, is about the same throughout the sequence. $M_{F} / M_{I}$ and the curve type indicate a lessening degree of maghemitization with increasing depth.

\section{Discussion of Hole 519A}

The flow of Lithologic Unit 4 in Hole 519A provides a good object for a magneto-petrological study, one that in the present case is supported by optical and electron microprobe analysis techniques. Some magnetic properties, such as Curie temperature and spontaneous magnetization, depend only on chemical composition; others, such as coercive force and remanence, depend on both chemical composition and microstructure. The characteristics of the inversion phenomenon depend on composition. The ratio $M_{R S} / M_{S}$ depends on grain size, although it is not entirely independent of composition.

Consider first those properties that depend on both composition (represented by $x$ and $z$ ) and microstructure (taken as being effective grain size). As depth in the flow increases, $N R M / M_{R S}$ and $\chi_{\mathrm{o}}$ rise, and hardness $\left(M D F, H_{c}\right)$ and $M_{R S} / M_{s}$ fall. In terms of microstructure this would be explicable in terms of an increasing effective grain volume with increasing depth, the grain size 
rising from the monodomain into the pseudo-single-domain range $(<1 \mu \mathrm{m}$ rising to $>10 \mu \mathrm{m})$. In terms of composition, the rise in $N R M / M_{R S}$ ratio would follow from a fall in $M_{S}$ at room temperature and a rise in $T_{B}$, the former indicating a rise in $x$ and $z$ and the latter a fall in $x$ and a rise in $z$. The hardness-related parameters suggest a decreasing $\mathrm{Fe}^{2+}$ concentration with increasing depth (i.e., a decreasing $x$ and/or an increasing $z$ ). The general inference, then, is an increase in volume, an increase in $z$, and a fall in $x$ with increasing depth.

Consider now those properties that are independent of microstructure. The rise in $M_{s}$ with increasing depth would suggest a decreasing $x$ and/or a decreasing $z$. The fall in $T_{c 1}$ would correspond to an increasing $x$ or a decreasing $z$. The inversion behavior $\left(T_{c 2}, M_{F} / M_{I}\right.$, and type of curve) clearly seems to indicate a decrease in $z$ with increasing depth.

To match up the two sets of properties it must be concluded that those properties that depend on both microstructure and composition are dominated by the variation in microstructure with depth (i.e., a rise in the effective grain size of the magnetic fraction) rather than by the variation in composition with depth. The composition-dependent properties (e.g., $M_{s}, T_{c}$ ) indicate a decreasing degree of maghemitization with depth, the $M_{F} / M_{I}$ values being consistent with a value of $z$ approaching unity. The value of $x$ may or may not vary with depth, but the value of $T_{c 1}$ at the lowest point sampled suggests that $x$ exceeds $\mathbf{0 . 6}$.

To summarize, the magneto-mineralogical implications of the magnetic properties are as follows. The higher part of the flow cooled more rapidly than the lower part. Low-temperature alteration is highly developed in the upper part and less so at greater depth in the flow, presumably because the smaller grains are more susceptible to alteration and the weathering medium is closer to the top of the flow. Rough quantitative estimates of grain size range, composition, and concentration (a few percent) are possible. The optical and microprobe data support these conclusions, although the observations are restricted to grains in the optical size range. One point of interest about the $\bar{x}$ and $\bar{z}$ values returned by the microprobe is that an increase in $\bar{x}$ may appear to accompany the fall in $\bar{z}$ that accompanies increasing depth. The values of $x$ and $z$ may be quite independent, of course, but if it is assumed that $x$ is uniform throughout the fresh flow, alteration would seem to have brought about a reduction in $x$. This would not be consistent with the presence of a submarine weathering mechanism (Petersen et al., 1979), because a leaching out of iron should produce an increase in $x$, although at least one other investigation suggests that $x$ decreases with age for submarine basalts (Steiner, 1981).

\section{PILLOWS AND FLOWS OF HOLE 522B}

\section{Optical and Electron Microprobe Analysis}

The results of the optical examination of the basement material from Hole 522B are summarized in Table 1. One striking feature is the absence of ilmenite from these samples. Sample 522B-3-4, $61 \mathrm{~cm}$, which is from a pillow, is very different from the others in both silicate and opaque mineralogy, showing a silicate texture that is associated with rapid quenching. The titanomagnetites occur as individual grains evenly dispersed throughout the matrix, and all samples from this site are generally fine grained and at the limit of the microprobe beam. They are subhedral to anhedral in form. Sample 522B-5-2, $30 \mathrm{~cm}$ stands out among the flows; it is apparently the most rapidly quenched of the samples studied.

Only Sample 522B-5-2, $75 \mathrm{~cm}$ contains sulfide spherules. They are very small and appear to be homogeneous pyrite. Samples 522B-4-1, $43 \mathrm{~cm}, 522 \mathrm{~B}-4-1,17 \mathrm{~cm}$, and 522B-3-4, $64 \mathrm{~cm}$ contain small amounts of subhedral/ skeletal pyrite grains.

The results of the microprobe analyses are listed in Table 2 for samples in which large enough grains of titanomagnetite/titanomaghemite could be found. Even in these cases the probability of beam spreading into the surrounding minerals is high. The average $x$ value from the various flows of Hole 522B is essentially similar to that of the Hole 519A flow, and the $z$ values are not significantly different.

\section{Intensity of $N R M$ and $M_{R S}$}

An upward trend in $N R M$ intensity with increasing depth may be indicated by the data (Table 3), but the samples are too few to draw more confident conclusions. Sample 522B-5-2, $30 \mathrm{~cm}$, which is from the lower flows, seems anomalous in having a low intensity. The deepest sample, Sample 522B-5-3, $41 \mathrm{~cm}$, which may be from a sill, has the highest intensity. The $M_{R S}$ values are fairly uniform throughout the sequence (except for Sample 522B-5-2, $30 \mathrm{~cm}$ ). The $N R M$ intensities are significantly higher than those of Hole 519A, whereas $M_{R S}$ is about the same. The $N R M / M_{R S}$ ratios of Hole 522B are therefore significantly higher than those of Hole 519A. The $M D F$ of $M_{R S}$ shows no trend with increasing depth and is of similar magnitude to that of Hole 519A, the pillow (Sample 522B-3-4, $61 \mathrm{~cm}$ ) and Sample 522B$5-2,30 \mathrm{~cm}$ being the "hardest" samples.

\section{Hysteresis Parameters and Initial Susceptibility}

The saturation magnetization, $M_{s}$ (listed in Table 3), shows no systematic change with position in the hole and is fairly uniform (again except for Samples 522B-5-2, $30 \mathrm{~cm}$ and 522B-3-4, $61 \mathrm{~cm}$, both of which have lower values than the rest of the set). Sample 522B-5-2, $30 \mathrm{~cm}$ is the hardest sample, with the highest $H_{c}$ value for all three holes and the highest $M_{R S} / M_{S}$, clearly in the monodomain range. Sample 522B-3-4, $61 \mathrm{~cm}$ is comparable to the hardest samples of Hole 519A. All other samples of Hole 522B are in the pseudo-single-domain/monodomain category.

Sample 522B-5-2, $30 \mathrm{~cm}$ has the lowest initial susceptibility of the samples from all three holes, but it has a fairly typical value of $\chi_{p}$. The pillow (Sample 522B-3-4, $61 \mathrm{~cm}$ ) also has a low initial value of susceptibility; it is similar to the values for the upper part of the flow of Hole 519A. The $Q$ values of samples from Hole 522B 
are generally higher than those from Holes 519A and 524 (see below) because of the low susceptibility; the NRM intensities are generally higher than in Hole 519A and of the same order as in Hole 524.

\section{Thermomagnetic Analysis}

$T_{c 1}$ and other temperatures were determined for some of the samples by using the magnetic balance; the results are shown in Table 4. Other samples were run in a lowfield susceptibility bridge, and $T_{c 1}$ only was determined for these samples. No systematic downhole variation in properties is apparent. The uppermost sample (Sample 522B-3-4, $61 \mathrm{~cm}$, pillow) is probably significantly maghemitized even though the $M_{F} / M_{I}$ ratio is low. The ratio may be low because the grains are small; the magnetic inversion product of even smaller grains is probably superparamagnetic. The ascending magnetization on the cooling curve does not therefore rise to a value that reflects the concentration of the magnetic phase. For such samples the determination of $M_{F} / M_{I}$ below room temperature may be a better diagnostic technique. Samples 522B-4-1, $17 \mathrm{~cm}$ and 522B-4-1, $43 \mathrm{~cm}$ are probably only slightly maghemitized. Part of Sample 522B-4-1, $108 \mathrm{~cm}$ was found to be relatively unaltered and part was highly altered, hence the two values of $T_{c 1}$ in the table. Sample 522B-5-2, $30 \mathrm{~cm}$ may be the most highly altered sample and has the highest $T_{c 1}$ and $M_{F} / M_{I}$ ratio. The flow sequence in Section 5-2 (30,75, 102, and $139 \mathrm{~cm})$ seems to show a reduction in maghemitization with increasing depth.

\section{Discussion of Basalts of Hole 522B}

The samples are distributed among a number of cooling units, and no systematic downhole variations are apparent. More closely spaced and comprehensive sampling may reveal rhythms in grain size and composition.

Sample 522B-3-4, $61 \mathrm{~cm}$ contains fine-grained monodomain $(<1 \mu \mathrm{m})$ titanomaghemites that are similar to the finer grained samples of the Hole 519A flow. The very fineness of this sample may tend to disguise its possibly high degree of maghemitization.

The magnetic minerals of Samples 522B-4-1, $17 \mathrm{~cm}$ and 522B-4-1, $43 \mathrm{~cm}$ have about the same composition and microstructure. This flow is coarser and less altered than the pillow (Sample 522B-3-4, $61 \mathrm{~cm}$ ).

Some of the properties within the flow represented by the three samples $522 \mathrm{~B}-5-2,30 \mathrm{~cm}, 522 \mathrm{~B}-5-2,75 \mathrm{~cm}$, and $522 \mathrm{~B}-5-2,139 \mathrm{~cm}$ do seem to vary systematically. Magnetic hardness ( $M D F$ and $H_{c}$ ) falls with depth (increasing grain size/decreasing $x$ /increasing $z$ ), and susceptibility rises. $M_{R S} / M_{S}$ falls (increasing grain size). $T_{c 1}$ falls, as does $M_{F} / M_{I}$. This flow seems therefore to have the same history as the flow of Hole 519A, but it probably has a lower overall grain size.

The lowest sample, 522B-5-3, $41 \mathrm{~cm}$, contains pseudo-single-domain grains (a few $\mu \mathrm{m}$ in size).

Insofar as the magnetic fraction could be studied by optical and electron microprobe analysis techniques, these observations are consistent with the conclusions of the magnetic study.

\section{SILLS OF HOLE 524}

\section{Optical and Electron Microprobe Analysis}

The results of the optical examination of the material from Hole 524 are summarized in Table 1. The opaque mineralogy is similar to that of Hole 519A, but no limonite or sulfide spherules were found and no alteration of the opaques is evident. The titanomagnetites are by far the most abundant oxides. The titanomagnetites are generally larger than those found in the fine-grained samples of Hole 522B and similar to those of the central and lower parts of Hole 519A, showing various welldefined outlines of the cubic system rather than the subhedral outlines of Hole 519A. Toward the chilled margin of the upper sill, Sample 524-29-2, $28 \mathrm{~cm}$ has relatively small grains. Some are skeletal, but the majority are subhedral or euhedral; presumably a large proportion of included silicate was trapped during rapid grain growth. Toward the center of the upper sill the grains become generally larger (with the exception of two veined samples, Samples 524-30-2, $52 \mathrm{~cm}$ and 524-30-2, 110 $\mathrm{cm})$ and have fewer inclusions. The skeletal grains are fewer but larger and better developed, exhibiting more geometric skeletal shapes. The central unit of the lower sill exhibits the same sequence of forms, from top to center, as the upper sill, but it has larger grains with more perfect euhedral forms, fewer inclusions, and some well-developed, clean-cut skeletal grains. Sample 524-33-3, $131 \mathrm{~cm}$, toward the base of the central unit, shows a decrease in grain size and associated increased inclusions in the euhedral/subhedral grains and less well developed skeletals. The veined rocks of Samples 524$30-2,52 \mathrm{~cm}$ and 524-30-2, $110 \mathrm{~cm}$ (upper sill) and 524$33-2,98 \mathrm{~cm}$ (lower central sill) have smaller grains than would be expected from their positions within the units.

Ilmenites decrease in abundance with increasing depth in the upper sill, with laths and subhedral grains becoming less common, noticeably so in the case of the laths. In the lower sill the only sample that contains ilmenite (and this in the form of laths) is Sample 524-33-2, 52 $\mathrm{cm}$, at the top.

No sulfide spherules occur in the Hole 524 samples, in contrast to Hole 519A. No pyrrhotite is found, but minor sulfides occur as pyrite within the main groundmass of the higher parts of the sills. Pyrite is abundant in the microfractures of Samples 524-30-2, $52 \mathrm{~cm}$, $524-30-2,110 \mathrm{~cm}$, and 524-33-2, $98 \mathrm{~cm}$.

The microprobe data for the titanomagnetites/titanomaghemites are listed in Table 2. The largest and apparently most homogeneous and least pitted grains were chosen for analysis. The $x$ values for the upper and lower sill are not significantly different. The upper sill may be the more highly oxidized, and one sample from the lower sill (Sample 524-33-3, $100 \mathrm{~cm}$ ) returned consistently low $z$ values.

\section{Intensity of Natural Remanent Magnetization and Isothermal Remanence}

The magnetic properties of the sills of Hole 524 are listed in Table 3. The NRM intensity increases with in- 
creasing depth in the upper sill, except for Sample $524-31-2,69 \mathrm{~cm}$, which is anomalously low. The values of $M_{R S}$ also suggest an increase with depth, although the lowest two samples have low values. $N R M / M_{R S}$ is approximately constant, apart from the lowest two samples. The values of NRM are an order of magnitude higher than the Hole 519A values, and $M_{R S}$ is slightly higher (if significantly different). The $N R M / M_{R S}$ ratios are higher than those of Hole 519A. The values of $M D F$ are an order of magnitude smaller than for Hole 519A. Hole 524 (upper sill) and Hole 519A have similar grain sizes and concentrations of magnetic oxides (at least in the optical range). Any significant differences must be due to the forms of the grains, differences in chemical composition, or alteration history. Hole 524 is magnetically softer but has the higher NRM.

In the lower sill no trends in $N R M$ intensity or $M_{R S}$ are evident, and the $N R M / M_{R S}$ ratios are quite scattered. Values of all three quantities, and $M D F$, are in the same range as for the upper sill.

\section{Hysteresis Parameters and Initial Susceptibility}

The saturation magnetization $\left(M_{s}\right)$ of this group of samples is significantly higher than that of either Hole 519A or Hole 522B (Table 3). The coercive force and $M_{R S} / M_{s}$ values of the upper sill fall at the lower end of the range observed for Hole $519 \mathrm{~A}$, and the susceptibilities are much higher, again indicating a magnetic hardness that is lower, although still in the pseudo-single-domain range. The $Q$ values seem to rise systematically with increasing depth in the upper sill and generally lie between those of Hole 519A and Hole 522B.

The lower sill has properties similar to those of the upper sill. The values of $H_{c}$ and $M_{R S} / M_{s}$ indicate that there may be a systematic fall in hardness with increasing depth.

\section{Thermomagnetic Analysis}

The results of thermomagnetic runs are listed in Table 4. As before, some of the samples were run on a high-field balance and some on a low-field bridge. The $T_{c 1}$ values and the curve types suggest that the upper sill may be more altered than the lower sill, with the upper sill most altered toward the bottom (in contrast to the flows of Holes 519A and 522B) and the lower sill more altered toward the top.

\section{Discussion of the Sills of Hole 524}

The $M_{s}$ values indicate that the concentration of magnetic mineral may be significantly higher than in Hole $519 \mathrm{~A}$ or 522B. The difference is not evident from optical examination and suggests that the sills of Hole 524 contain a higher concentration of stable monodomain magnetic minerals in the submicroscopic range than the other holes. A possible slight decrease in effective grain size with increasing depth may be indicated in both the upper and lower sills, the grains being in the pseudo-single-domain range (a few $\mu \mathrm{m}$ ). However, the microstructure-sensitive magnetic parameters are more uniform in the sills than in the flows. The sill samples are not as highly altered as the more highly maghemitized flow samples, and it may be that the sediment separating the upper and lower sills acted as a conduit for the weathering medium, producing a greater degree of alteration in the base of the upper sill and the top of the lower sill.

\section{CONCLUSIONS AND COMPARISON OF BASALTIC SAMPLES FROM HOLES 519A, 522B, AND 524}

The mean NRM intensities (and standard deviations) of the basalts from Holes 519A, 522B, and 524 are 4.46 $\times 10^{-4}\left(1.52 \times 10^{-4}\right), 1.86 \times 10^{-3}\left(1.07 \times 10^{-3}\right)$, and $1.94 \times 10^{-3}\left(1.43 \times 10^{-3}\right) \mathrm{Am}^{2} / \mathrm{kg}$, respectively. The youngest material therefore has the lowest remanence intensity. The average $Q$ ratios are $12(6), 26(13)$, and 7 (5). The stable inclinations are $+7^{\circ}\left(6^{\circ}\right),+32^{\circ}\left(34^{\circ}\right)$, and $+5^{\circ}\left(60^{\circ}\right)$, respectively. The number of samples is, of course, too small to drawn any conclusion about the contribution of this material to changes in the pattern of geomagnetic anomalies. The number of samples of like material (i.e., sills and flows) is even smaller. The sills are the softest of the materials studied and have the highest $M_{s}$ and therefore the largest concentration of well-developed magnetic mineral grains. The sills of Hole 524 are probably the least altered of the basalts examined. No significant differences in degree of alteration were found between the flows of Holes 519A and 522B, but systematic variations were found locally. In general, those magnetic properties that are dependent on both composition and microstructure seem to be dominated by the effects of microstructure.

The flow of Hole 519A is highly maghemitized at the top, the degree of maghemitization decreasing with depth in the flow. The effective grain size increases with increasing depth. The samples of Hole 522B are distributed among a number of cooling units, and no systematic downhole variations are apparent. Variations within units are however consistent with the cooling/alteration sequence that might be expected within the units. The upper and lower sills of Hole 524 have a larger concentration of well-developed magnetic mineral grains than the pillows and flows of Holes 519A and 522B. Maghemitization seems to have developed from the boundaries of the sills in contact with the sediments separating the upper and lower sills.

\section{ACKNOWLEDGMENTS}

One of the authors (P.T.) has been in receipt of a Natural Environment Research Council (NERC) postdoctoral fellowship and another (J.H.) is in receipt of a NERC postgraduate studentship. We thank Mr. W. T. Davison for help with the microprobe measurements and our referees, Dr. P. W. Readman (Dublin) and Dr. R. Thompson (Edinburgh). We also want to thank Miss Stella Sprake for help with the computations.

\section{REFERENCES}

Day, R., 1977. TRM and its variation with grain size. J. Geomagn. Geoelectr., 29:233-265.

Néel, L., 1949. Theorie du trainage magnetique des ferromagnétiques en grains fins avec applications aux terres cuites. Ann. Geophys., 5:99-136.

Özdemir, O., and O'Reilly, W., 1981. Laboratory synthesis of aluminum-substituted titanomaghemites and their characteristic properties. J. Geophys., 49:93-100. 
1982. An experimental study of thermoremanent magnetization acquired by synthetic monodomain titanomaghemites. $J$. Geomagn. Geoelectr., 34:467-478.

Petersen, N., Eisenach, P., and Bleil, U., 1979. Low temperature alteration of the magnetic minerals in ocean floor basalts. In Talwani, M., Harrison, C. G., and Hayes, D. B. (Eds.), Deep Drilling Results in the Atlantic Ocean: Ocean Crust: Am. Geophys. Union (Washington), pp. 210-229.
Ryall, P. J. C., Hall, J. M., Clark, J., and Milligan, T., 1977. Magnetization of oceanic crustal Layer 2-results and thoughts after DSDP Leg 37. Can. J. Earth Sci., 14:684-706.

Steiner, M., 1981. Ti loss during low temperature oxidation. IAGA Bull., 45:224. (Abstract)

Wasilewski, P. J., 1968. Magnetization of ocean basalts. J. Geomagn. Geoelectr., 20:129-154. 